This item was submitted to Loughborough's Research Repository by the author.

Items in Figshare are protected by copyright, with all rights reserved, unless otherwise indicated.

\title{
Rabi-coupled two-component Bose-Einstein condensates: Classification of the ground states, defects, and energy estimates
}

PLEASE CITE THE PUBLISHED VERSION

http://dx.doi.org/10.1103/PhysRevA.94.023616

\section{PUBLISHER}

(C) American Physical Society

\section{VERSION}

AM (Accepted Manuscript)

\section{PUBLISHER STATEMENT}

This work is made available according to the conditions of the Creative Commons Attribution-NonCommercialNoDerivatives 4.0 International (CC BY-NC-ND 4.0) licence. Full details of this licence are available at: https://creativecommons.org/licenses/by-nc-nd/4.0/

\section{LICENCE}

CC BY-NC-ND 4.0

\section{REPOSITORY RECORD}

Aftalion, Amandine, and Peter Mason. 2019. "Rabi-coupled Two-component Bose-einstein Condensates: Classification of the Ground States, Defects, and Energy Estimates". figshare. https://hdl.handle.net/2134/22494. 


\title{
Rabi-coupled two-component Bose-Einstein condensates: classification of the ground states, defects and energy estimates
}

\author{
Amandine Aftalion ${ }^{1} \&$ Peter Mason $^{2}$ \\ ${ }^{1}$ CNRS \&S Université Versailles-Saint-Quentin-en-Yvelines, \\ Laboratoire de Mathématiques de Versailles, CNRS UMR 8100, \\ 45 avenue des États-Unis, 78035 Versailles Cédex, France \\ ${ }^{2}$ Department of Physics, Loughborough University, Loughborough, LE11 3TU, United Kingdom
}

\begin{abstract}
We classify the ground states and topological defects of two-component Bose-Einstein condensates under the effect of internal coherent Rabi coupling. We present numerical phase diagrams which show the boundaries between symmetry breaking components and various vortex patterns (triangular, square, bound state between vortices). We estimate the Rabi energy in the Thomas-Fermi limit which allows us to have an analytical description of the point energy leading to the formation of the various vortex patterns.
\end{abstract}

PACS numbers: 03.75.Mn,03.75.Lm,67.85.Fg

\section{INTRODUCTION}

The realization of multi-component Bose-Einstein condensates $[1,2]$ provides opportunities to explore the rich physics brought about by the spin degrees of freedom. For instance, there have been studies of spin waves and phase separation [3], macroscopic quantum tunneling [4], Rabi oscillations [5], the coupling between spin gradients and superfluid flow, squeezed spin states, spinor condensates $[6,7]$, vortices and other topological excitations $[8,9]$.

Theoretically and numerically, there have been many studies of the ground-state properties of two-component condensates [10-19]. We see that, for instance, as in single component condensates, rotation induces vortices: these vortices can nucleate in either or both components and their interactions lead to the formation of vortex lattices and vortex molecules. Such vortex molecules have been studied in multi-component condensates: fractional vortices in different component with fractional circulations can form many different patterns [20-23]. For instance, as the intercomponent coupling is varied, one can go from a triangular lattice of vortices, to a square lattice or vortex sheets.

In addition to the intercomponent coupling between different components, we can have a spin-orbit coupling, a dipolar coupling, or indeed a one-body coherent Rabi coupling. The latter coupling provides the same kind of interactions as a Josephson coupling in superconductors. It is the effect of the coupling and the interplay between rotation and intercomponent coupling, that we will focus on in this paper. Specifically we are interested in the vortex lattices that have been numerically studied in $[24,25]$; these authors were able to see the appearance of multidimer bound states. This is a state whereby a pair of vortices (nucleated by the rotation) of different components 'bind' together as a 'molecule'. These molecules then interact in a non-trivial manner with other molecules, with the result that a rich hier- archy of patterns, such as honeycombed, triangular and square, are formed by the molecules. We want to analyze these patterns more precisely in this paper, performing numerical simulations over a wide parameter space (to include intercomponent couplings, rotation frequencies and Rabi frequencies) and further to analyse from an analytical perspective the critical parameters that govern their appearance.

\section{PROBLEM STATEMENT AND ENERGY FUNCTIONAL}

In the mean-field zero temperature limit, the rotating two-dimensional two-component condensate with Rabi coupling is described by the following non-dimensional energy functional in terms of the wave functions $\psi_{1}$ and $\psi_{2}$

$$
\begin{aligned}
E= & \int \sum_{k=1}^{2}\left(\frac{1}{2}\left|\nabla \psi_{k}\right|^{2}+\frac{1}{2} r^{2}\left|\psi_{k}\right|^{2}-\Omega \psi_{k}^{*} L_{z} \psi_{k}+\frac{g N}{2}\left|\psi_{k}\right|^{4}\right) \\
& +g_{12} N\left|\psi_{1}\right|^{2}\left|\psi_{2}\right|^{2}-\omega\left(\psi_{1}^{*} \psi_{2}+\psi_{1} \psi_{2}^{*}\right) \quad d^{2} r
\end{aligned}
$$

with time, energy and length measured in units of $\tilde{\omega}^{-1}$, $\hbar \tilde{\omega}$ and $\sqrt{\hbar / m \tilde{\omega}}$ respectively, $\tilde{\omega}$ being the trapping frequency. Equation (1) is subject to the constraint that the total number of particles is conserved: $\int\left|\psi_{1}\right|^{2}+\left|\psi_{2}\right|^{2}=1$. There are a number of parameters introduced: $g$ is the self interaction of each component (intracomponent coupling) that we have taken to be equal and positive for both components, $g_{12}$ measures the effect of interaction between the two components (intercomponent coupling), $N$ is the total atom number and $\Omega$ is the frequency of rotation, applied equally to both components. The angular momentum operator acting in the $z$ direction is $L_{z}=-i\left(x \partial_{y}-y \partial_{x}\right)$. The final term of Eq. (1) represents the coherent (one-body) Rabi coupling of atomic internal 
states where $\omega$ denotes the Rabi frequency, again taken to be identical for both components. The ground state and excited states have been studied in [26] and the different vortex patterns in $[24,25]$.

It is helpful to express the above energy functional in terms of a non-linear Sigma formalism. General details of this formalism can be found elsewhere (see [14] for example). We write the energy in terms of the total density $\rho$,

$$
\rho=\left|\psi_{1}\right|^{2}+\left|\psi_{2}\right|^{2}
$$

and define a normalised complex-valued spinor $\chi=$ $\left[\chi_{1}, \chi_{2}\right]^{T}$ so that wave functions are decomposed as $\psi_{1}=$ $\sqrt{\rho} \chi_{1}$ and $\psi_{2}=\sqrt{\rho} \chi_{2}$. The spin density is $\boldsymbol{S}=\bar{\chi} \boldsymbol{\sigma} \chi$, where $\boldsymbol{\sigma}=\left(\sigma_{x}, \sigma_{y}, \sigma_{z}\right)$ are the Pauli matrices, so that the components of $\boldsymbol{S}$ are

$$
\begin{aligned}
& S_{x}=\chi_{1}^{*} \chi_{2}+\chi_{2}^{*} \chi_{1}, \\
& S_{y}=-i\left(\chi_{1}^{*} \chi_{2}-\chi_{2}^{*} \chi_{1}\right), \\
& S_{z}=\left|\chi_{1}\right|^{2}-\left|\chi_{2}\right|^{2},
\end{aligned}
$$

and $|\boldsymbol{S}|^{2}=1$. For a rotating condensate, it is natural to introduce $\Theta_{k}$ the phase of $\psi_{k}$, that is $\psi_{k}=\sqrt{\rho}\left|\chi_{k}\right| e^{i \Theta_{k}}$, $\Theta=\Theta_{1}+\Theta_{2}$, and $\boldsymbol{R}=S_{y} \nabla S_{x}-S_{x} \nabla S_{y}$ so that

$$
\boldsymbol{v}_{\mathrm{eff}}=\frac{\nabla \Theta}{2}+\frac{\boldsymbol{R} S_{z}}{2\left(1-S_{z}^{2}\right)}=\frac{1}{2} S_{z} \nabla\left(\Theta_{1}-\Theta_{2}\right) .
$$

This allows us to rewrite the energy functional (1) as

$$
\begin{aligned}
E=\int & \frac{1}{2}(\nabla \sqrt{\rho})^{2}+\frac{\rho}{8}(\nabla \boldsymbol{S})^{2}+\frac{\rho}{2}\left(\boldsymbol{v}_{\mathrm{eff}}-\boldsymbol{\Omega} \times \boldsymbol{r}\right)^{2} \\
& +\frac{1}{2}\left(1-\Omega^{2}\right) r^{2} \rho+\frac{\rho^{2}}{2}\left(c_{0}+c_{2} S_{z}^{2}\right)-\rho \omega S_{x} \quad d^{2} r
\end{aligned}
$$

where, defining $\delta=g_{12} / g$,

$$
\begin{aligned}
& c_{0}=\frac{N}{2}\left(g+g_{12}\right)=\frac{g N}{2}(1+\delta), \\
& c_{2}=\frac{N}{4}\left(g-g_{12}\right)=\frac{g N}{2}(1-\delta) .
\end{aligned}
$$

Note that overall stability is maintained provided $\delta>-1$. In the following sections, we will first present numerically obtained phase diagrams which show the various ground state profiles for the two-components (Sect. III) and then move on to analytically analyse details of these profiles (Sect. IV).

\section{DESCRIPTION OF THE PHASE DIAGRAMS}

In this section, we present numerically obtained phase diagrams for the ground state profiles that result from the energy functional of Eq. (1). The simulations are con- ducted on the coupled Gross-Pitaevskii equations that follow from the variation $i \partial \psi_{k} / \partial t=\delta E / \delta \psi_{k}^{*}$, for $k=1,2$ :

$$
\begin{aligned}
i \frac{\partial \psi_{k}}{\partial t}=- & \frac{1}{2} \nabla^{2} \psi_{k}+\frac{1}{2} r^{2} \psi_{k}-i \Omega\left(y \frac{\partial \psi_{k}}{\partial x}-x \frac{\partial \psi_{k}}{\partial y}\right) \\
& +g N\left|\psi_{k}\right|^{2} \psi_{k}+g_{12} N\left|\psi_{3-k}\right|^{2} \psi_{k}-\omega \psi_{3-k}
\end{aligned}
$$

The ground state of the above coupled Gross-Pitaevskii equations for a given parameter set can be numerically solved by propagating initial wave functions for the two different components in imaginary time $(t \rightarrow-i t)$ and subject to the normalisation of the total particle number (to 1). Random noise is added to these initial wave functions in order to break any residual symmetries, and the system will over imaginary time relax to a local ground state. Simulations are performed in Fortran using a pseudospectral split-step method. The presence of many vortices in the system means that it is not always possible to find the true minimizing energy state, however the use of various initial data converging to the same, or similar, state allows us to say with confidence that the true ground state will be close to the ones we present.

We use the following values of parameters: $g=1$, $N=10^{4}$, together with $\Omega \in[0,1), \omega \geq 0$ and $\delta \geq 0$. We preserve the constraint $\int\left|\psi_{1}\right|^{2}+\left|\psi_{2}\right|^{2}=1$. These parameters place us in the Thomas-Fermi regime (see Sect. IV for further details), and furthermore are consistent with experimentally realised two-component condensates. As an example, we quote two papers that both consider a ${ }^{87} \mathrm{Rb}^{87} \mathrm{Rb}$ mixture with one isotope in spin state $\left|F=2, m_{f}=1\right\rangle$ and the other in state $|1,1\rangle[3,9]$, such that the masses and transverse trapping potentials are equal. The scattering lengths for each component are very close to one another in value, given by $a_{1}=53.35 \AA$ and $a_{2}=56.65 \AA$. In this paper we consider the scattering lengths to be equal, and so if we set it to be the average of these two quoted scattering lengths, we find (see [14] for calculation details) that $g N$ lies between 800 and $3 \times 10^{4}$, depending on the total number of atoms in the condensate. If Rabi coupling was added to these experiments, one could hope to observe the vortex patterns described below.

For each our of chosen parameter sets, we classify the ground state according to the densities, $\left|\psi_{k}\right|^{2}$, and the spin densities, $\boldsymbol{S}$. We break our analysis into three sections; no rotation, $\Omega$ small and $\Omega$ close to 1 .

\section{A. Zero rotation, $\Omega=0$}

In the case $\Omega=0$, the components do not display any phase $\left(\Theta_{1}=\Theta_{2}=0\right)$, which implies that we always have $S_{y}=0$. If additionally $\omega=0$, then $\delta=1$ separates the region of coexistence $(\delta<1)$ and segregation $(\delta>1)$.

For $\omega$ non zero, two different ground state profiles are 


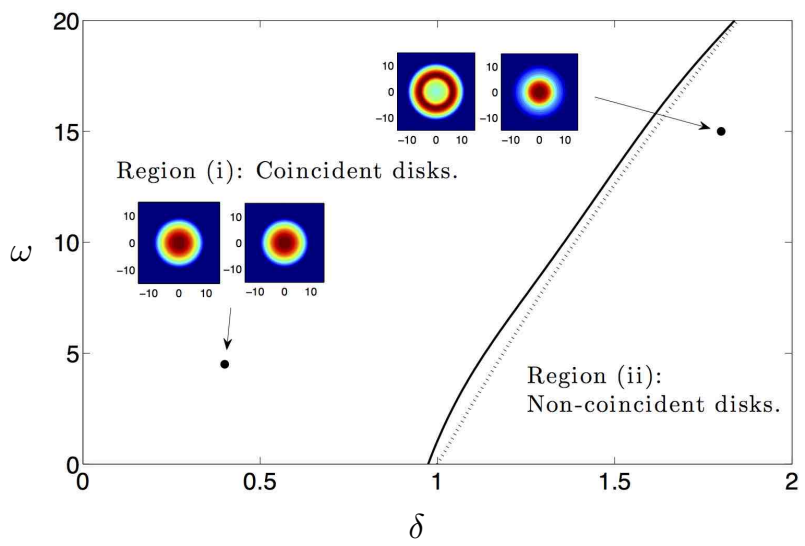

FIG. 1: (Color online) $\omega-\delta$ phase diagram with $\Omega=0$ describing the ground states of the energy functional (1). The numerical parameters are taken as $g=1$ and $N=10^{4}$. There are two identified regions: (i) two coincident disks and (ii) non-coincident disks. The regions are separated by a numerically (solid) and analytically (dotted; see Eq. (21)) determined boundary line. Typical density plots for each component (left panels, component 1 and right panels, component 2 ) are shown, with the numerical values correspond to: (i) $(\delta, \omega)=(0.4,4.5)$ and (ii) $(1.8,15)$.

present, as is seen in the phase diagram of Fig. 1. In region (i), there are two coincident disks and $\psi_{1}=\psi_{2}$, which implies that $S_{z}=0$. In region (ii), the ground state profile is composed of non-coincident components: both components are disks, however one component has a global density maximum at the origin while the other component has a non-zero minimum of density at the origin. The density profiles of Fig. 2(a) exemplify this; there are two parts, an outer one where the wave functions of both components coincide and an inner one where they differ (the boundary between these two sections occurs when $r \approx 6.25)$. When $\delta$ gets large, the outer region gets very small, and the modulus of the second wave function gets very small, yet the components remain disks. When $\delta>1$ and $\omega$ is small, one of the wave functions is very small, yet always non zero.

Throughout region (ii), the total density remains an inverted parabola; see Fig. 2(a). The boundary between regions (i) and (ii) can be numerically determined and is shown on the phase diagram plot of Fig. 1 as a solid line. In Sect. IV, we will perform a simple Thomas-Fermi analysis to obtain the density and spin density profiles, as well as the boundary between regions (i) and (ii) (dotted line in Fig. 1; see Sect. IV.A).

\section{B. Low rotation, $\Omega=0.3$}

The rotation frequency, $\Omega=0.3$, is chosen such that a few vortices are nucleated in both components. The phase diagram in this case is shown in Fig. 3. The regions
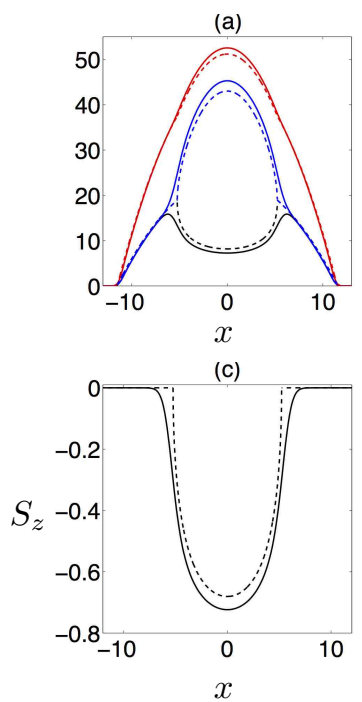

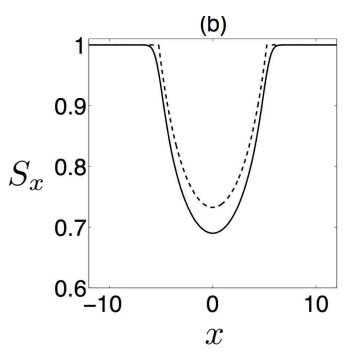

(d)

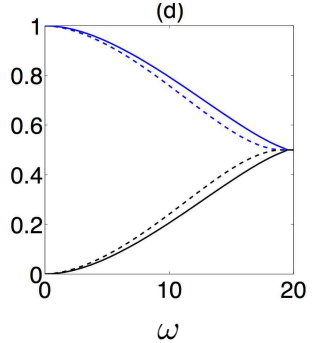

FIG. 2: (Color online) Example ground state profiles for region (ii) of the $\omega-\delta$ with $\Omega=0$ phase diagram of Fig. 1 showing (a) the individual density profiles, $\left|\psi_{1}\right|^{2}$ (black) and $\left|\psi_{2}\right|^{2}$ (blue), the total density $\rho$ (red), (b) $S_{x}$, (c) $S_{z}$ and (d) the number densities $N_{1}$ (black) and $N_{2}$ (blue). Solid lines indicate numerical simulations while dashed lines correspond to analytical estimates calculated through Eq.'s (11a) and (15b) (for $\rho$ ), Eq. (15a) (for $S_{x}$ ) and $S_{x}^{2}+S_{z}^{2}=1$ (for $S_{z}$ ) and $\left|\psi_{1}\right|^{2}=\left(1-S_{z}\right) \rho / 2\left(\right.$ for $N_{1}$, with $\left.1=N_{1}+N_{2}\right)$. The profiles are taken at $(\delta, \omega)=(1.8,15)$, and for (a-c) along $y=0$. The densities are multiplied by $N$ in (a).

of the phase diagram are defined as (i-a): two coincident disks with coincident vortices, (i-b) two coincident disks with non-coincident vortices, (ii) non-coincident disks with vortices and (iii) symmetry breaking components.

In regions (i-a) and (i-b), the ground states are coincident disks with vortices nucleated in both components; we separate these regions by determining whether the vortices are coincident (i-a) or non-coincident (i-b). In both regions, a vortex in one of the components is uniquely associated with a vortex in the other component. In this way, a 'vortex molecule' [24] is formed. The separation of the two vortex cores in each molecule is seen on the phase diagram to be dependent on both the interaction strength $\delta$ and the Rabi frequency $\omega$. When the vortices are non-coincident, then each vortex induces a peak of density in the other component (a vortex-peak, or coreless vortex structure; see $[14,15])$. As the vortex cores come closer together then the size of the peak is reduced and is evidently not present when the vortices coincide. In Fig. 4 we plot examples of the density profiles of each component in both regions, as well as a plot of the $S_{x}$ and $S_{z}$ components of the spin-density. Notice that in region (i-a), since both the disks and vortices are coincident, we have $\psi_{1}=\psi_{2}$ everywhere which immediately gives $S_{x}=1$ with $S_{y}=S_{z}=0$. In contrast, in region (ib), since the vortices are non-coincident, we have no such 


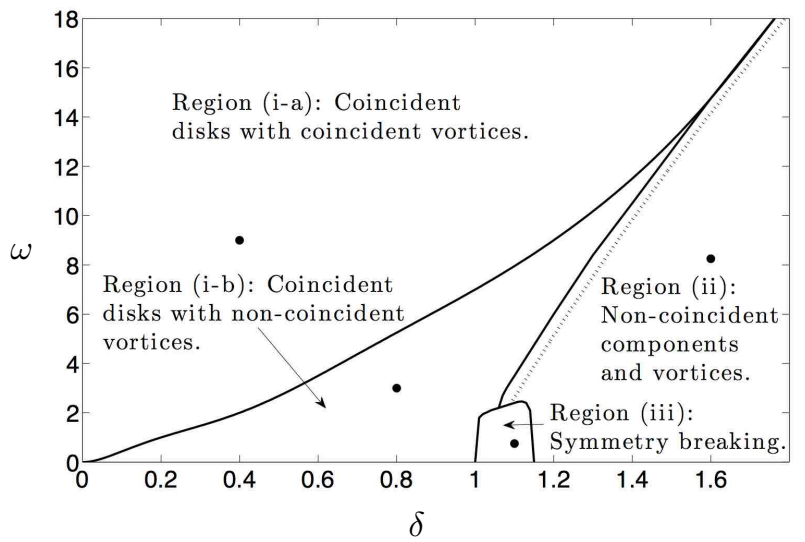

FIG. 3: $\omega-\delta$ phase diagram with $\Omega=0.3$ describing the ground states of the energy functional (1). The numerical parameters are taken as $g=1$ and $N=10^{4}$. There are three plus one identified regions: (i-a) two coincident disks with coincident vortices, (i-b) two coincident disks with noncoincident vortices, (ii) non-coincident disks and (iii) symmetry breaking components. The regions are separated by numerically (solid) and analytically (dotted; see Eq. (21)) determined boundary lines. Typical density and spin-density plots for each region are shown in Figs. 4-5, and correspond to the dots in the figure.

global condition, however we can say that $\psi_{1}=\psi_{2}$ away from the vortex molecule (see Fig. 4). Analysis of the separation of the vortex cores will be considered in more detail in Sect. IV.B where we will show that the vortexpeak structure inherent to two-component condensates without Rabi frequency (i.e. $\omega=0$ ) is crucial in order to understand the forms of $S_{x}$ and $S_{z}$ in region (i-b), and thus the separation of the vortex cores.

In regions (ii) and (iii) we have two non-coincident disks with non-coincident vortices and symmetry breaking components, respectively. Example density profiles from these two regions are shown in Fig. 5.

\section{High rotation, $\Omega=0.9$}

Our final example case takes a high rotation frequency $\Omega=0.9$. The phase diagram in this case is shown in Fig. 6(a) and contains the same three main regions: (i) coincident disks, (ii) noncoincident components and (iii) symmetry breaking. Region (i) consists of two coincident disks and can again be subdivided into regions (i-a): two coincident disks with coincident vortices, and region (i-b): two coincident disks with non-coincident vortices. However, in this high rotation case in which many vortices have been nucleated and well-formed vortex lattices are present, the non-coincident vortices of region (i-b) are seen to form distinct patterns in both the density profiles and the spin-density profiles. This has been analyzed in [23-25]. We thus further divide region (i-b) into regions
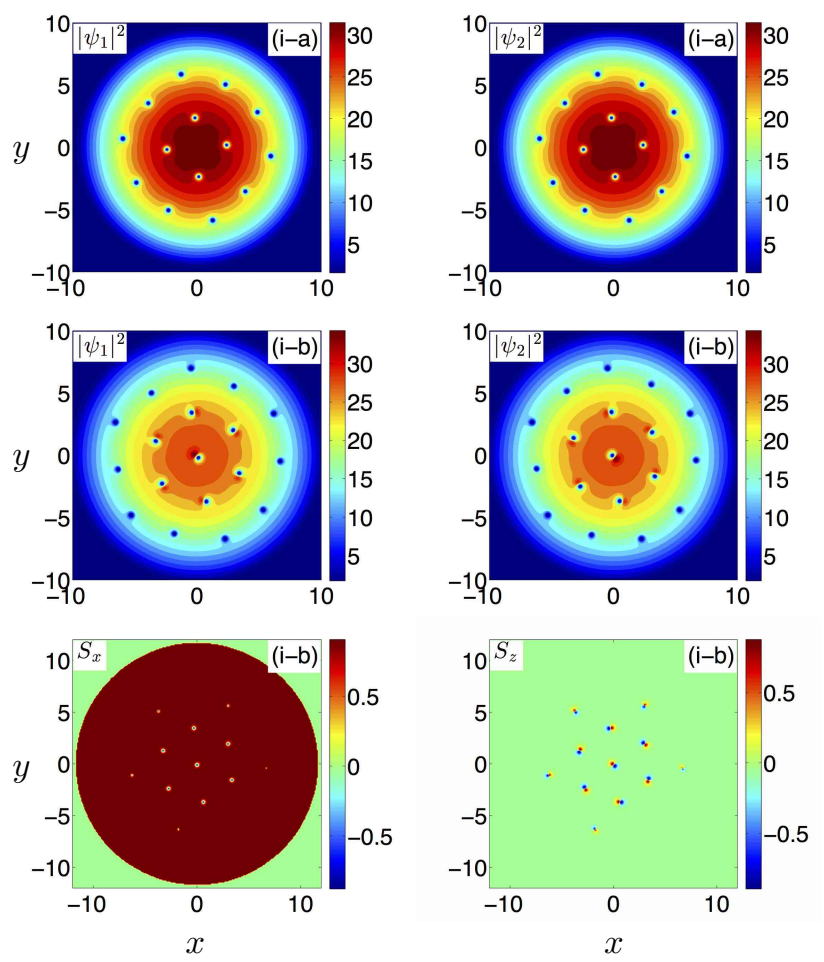

FIG. 4: (Color online) Example ground state profiles for regions (i-a) and (i-b) of the $\omega-\delta$ with $\Omega=0.3$ phase diagram of Fig. 3. Shown are the density profiles of component 1 and component 2 for regions (i-a) and (i-b) and $S_{x}$ and $S_{z}$ for region (i-b). The profiles for region (i-a) are taken at $(\delta, \omega)=(0.4,9)$ and for region (i-b) at $(0.8,3)$. The densities are multiplied by $N$.
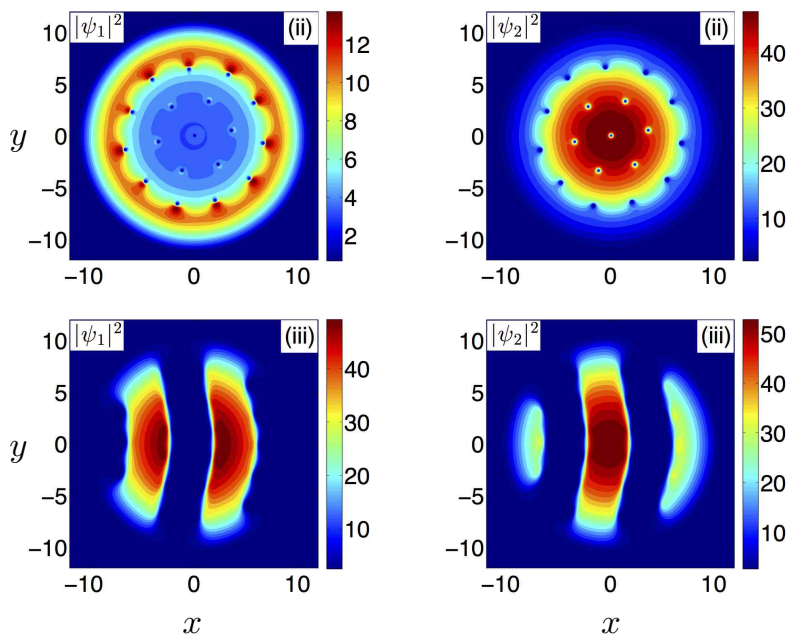

FIG. 5: (Color online) Example ground state density profiles for regions (ii) and (iii) of the $\omega-\delta$ with $\Omega=0.3$ phase diagram of Fig. 3. The profiles for region (ii) are taken at $(\delta, \omega)=(1.6,8.25)$ and for region (iii) at $(1.1,0.75)$. The densities are multiplied by $N$. 

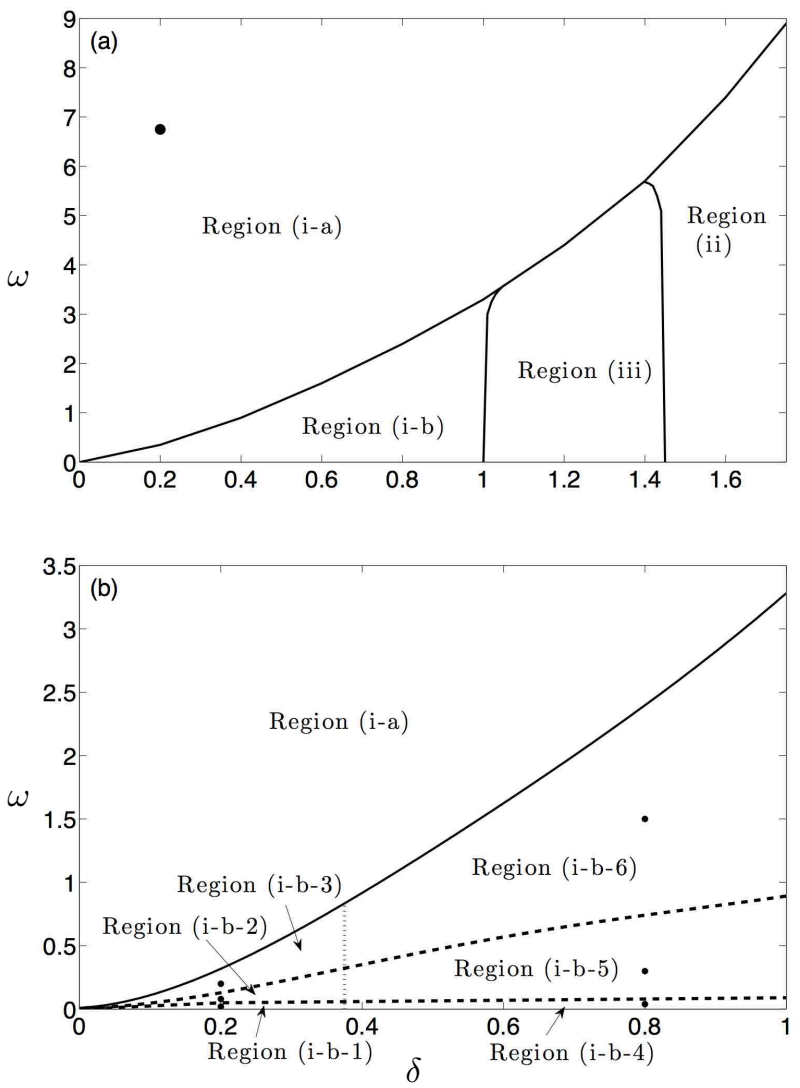

FIG. 6: (a) $\omega-\delta$ phase diagram with $\Omega=0.9$ describing the ground states of the energy functional (1). The numerical parameters are taken as $g=1$ and $N=10^{4}$. There are three plus one identified regions: (i-a) two coincident disks with coincident vortices, (i-b) two coincident disks with noncoincident vortices, (ii) non-coincident disks and (iii) symmetry breaking components. The regions are separated by numerically determined boundary lines. (b) Same as (a) but focusing on the six subregions of region (i-b) that exist when $\delta<1$. The six regions are separated by numerically determined dashed lines. Typical density and spin-density plots for regions (i-b-1)-(i-b-6) are shown in Figs. 7-10, and correspond to the dots in the figures.

(i-b-1)-(i-b-6) as indicated in Figure 6(b). We will now discuss these patterns in detail.

Regions (i-b-1)-(i-b-3) are plotted in Figs. 7 and 8. The general defining feature of these three regions is that the individual density profiles have triangular vortex lattices (see Figs. 7(a,b) and 8), however the patterns that the vortex molecules form - which can be clearly seen in plots of the total density $\rho$ and the $S_{x}$ component of the spin-density - change as we move around the phase diagram. These changes are seen in Figs. $7(\mathrm{c}, \mathrm{d})$ and 8. In region (i-b-1), the vortex molecules form regular hexagonal patterns, in region (i-b-2) they group together as pairs without any particular regular arrangement, and in region (i-b-3) they are individually well-separated and arranged on a regular triangular lattice.
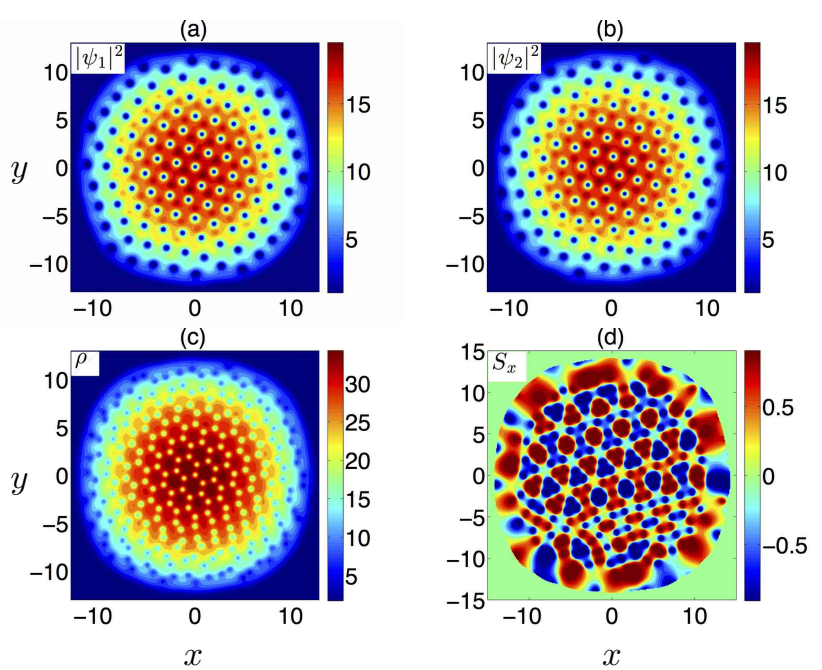

FIG. 7: (Color online) Example ground state profiles for region (i-b-1) of the $\omega-\delta$ with $\Omega=0.9$ phase diagram of Fig. 6 showing (a) $\left|\psi_{1}\right|^{2}$, (b) $\left|\psi_{2}\right|^{2}$, (c) $\rho$ and (d) $S_{x}$. The profile is taken at $(\delta, \omega)=(0.2,0.02)$. The densities are multiplied by $N$ in (a-c).

A result from two-component condensates in the absence of a Rabi coupling [13], and for large rotation, gives the value of $\delta \approx 0.38$ at which there is a transition from triangular vortex lattices to square vortex lattices. We find that, even when a Rabi coupling is included into the problem, deviation from this condition is small. The dotted line on Fig. 6 depicts this transition, and separates out regions (i-b- 1 )-(i-b-3), which exist for $0<\delta \lesssim 0.38$ from regions (i-b-4)-(i-b-6).

Regions (i-b-4)-(i-b-6) are plotted in Figs. 9 and 10. They exist for $0.38 \lesssim \delta<1$ with the general defining feature that the individual density profiles have square vortex lattices (see Figs. 9(a,b) and 10). As with regions (i-b-1)-(i-b-3), we find that the vortex molecules in regions (i-b-4)-(i-b-6) form interesting patterns, as is clearly seen in Figs. 9(c,d) and 10. In region (i-b-4), the vortex molecules form regular square patterns, in region (i-b-5) they group together as pairs retaining an overall regular arrangement, and in region (i-b-6) they are individually well-separated and arranged on a regular triangular lattice.

In region (i-a), see Fig. 11, the vortex lattices in $\left|\psi_{1}\right|^{2}$, $\left|\psi_{2}\right|^{2}$ and $\rho$ are all triangular, and as explained before, $S_{x}=1, S_{y}=S_{z}=0$. In Sect. IV.C we look to analytically understand all of these vortex lattice structures of regions (i-a) and regions (i-b-1)-(i-b-6).

Finally, the $\Omega=0.9$ phase diagram contains a significant symmetry breaking region, region (iii). The extent of this region can be directly compared to that when $\Omega=0.3$ (Fig. 3), however the density profiles for both the small and large rotation frequencies are much the same. We can see this by comparing a typical plot of a 

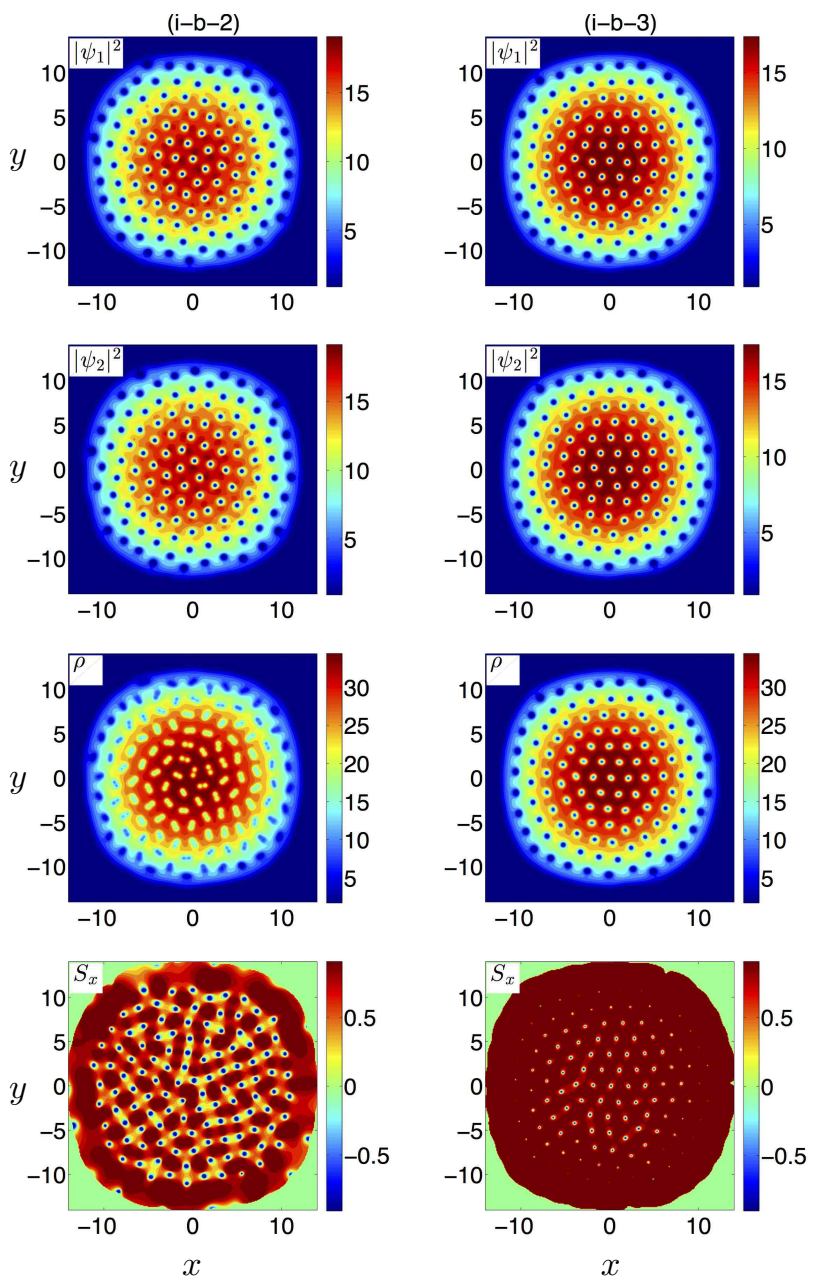

FIG. 8: (Color online) Example ground state profiles for regions (i-b-2) (left panels) and (i-b-3) (right panels) of the $\omega-\delta$ with $\Omega=0.9$ phase diagram of Fig. 6 showing $\left|\psi_{1}\right|^{2},\left|\psi_{2}\right|^{2}$, $\rho$ and $S_{x}$. The profiles are taken at $(\delta, \omega)=(0.2,0.08)$ for region (i-b-2) and $(0.2,0.2)$ for region (i-b-3). The densities are multiplied by $N$.

symmetry breaking state in the $\Omega=0.9$ phase diagram, shown in Fig. 12 with that obtained when $\Omega=0.3$ (Fig. $5)$. The difference lies in the number of stripes and the serpentine profile that appear; notice how the structures are reminiscent of the vortex sheet profiles seen in twocomponent rotating condensates in the absence of a Rabi coupling term $[14,27]$.

In these cases where there is an absence of a Rabicoupling, symmetry breaking occurs when $\delta>1$ : components are phase separated apart from a small interface layer that occurs at low densities. When a Rabi-coupling is introduced we see that the instability condition depends on both $\delta$ and $\omega$; however the symmetry breaking region of Fig. $6(\mathrm{a})$ is banded between $1 \lesssim \delta \lesssim 1.42$, and remains practically independent of the value of $\omega$, until $\omega$ becomes large enough at which point the symmetry
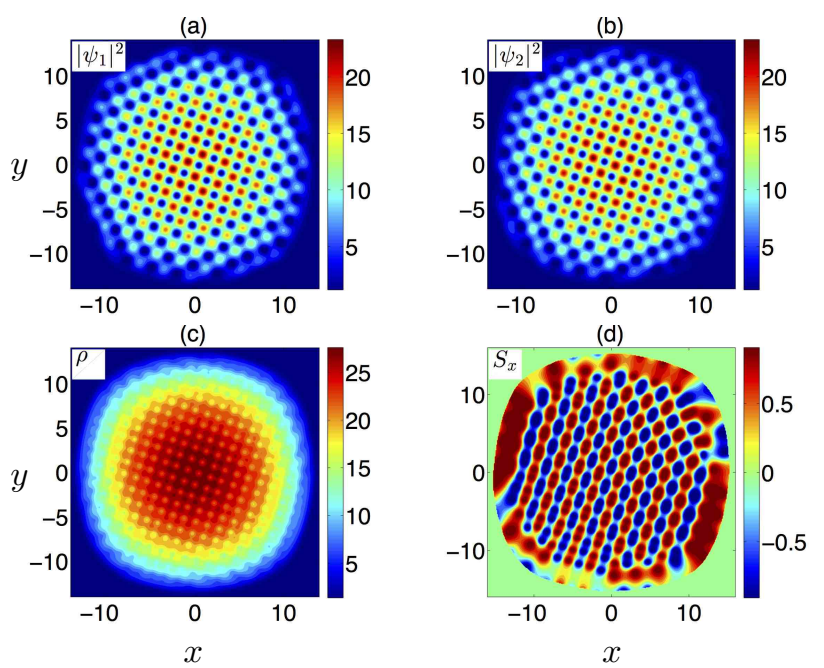

FIG. 9: (Color online) Example ground state profiles for region (i-b-4) of the $\omega-\delta$ with $\Omega=0.9$ phase diagram of Fig. 6 showing (a) $\left|\psi_{1}\right|^{2}$, (b) $\left|\psi_{2}\right|^{2}$, (c) $\rho$ and (d) $S_{x}$. The profile is taken at $(\delta, \omega)=(0.8,0.04)$. The densities are multiplied by $N$ in (a-c).

breaking state is no longer the preferred state since the Rabi term dominates the coupling term and coexistence is possible. The implication here is that the instability condition, while dependent on both $\delta$ and $\omega$, is in some regions of phase space, dominated by the interplay between the rotation and the coupling strengths.

It is at this point that it is interesting to make a comparison with the work of Cipriani \& Nitta [24]. They considered a fast rotating Rabi-coupled condensate $(\Omega=$ 0.98 ), producing a $\omega / \delta-\delta$ phase diagram (instead of the $\omega-\delta$ phase diagrams that we have shown up to now in this paper). We have thus rescaled the vertical axis of the phase diagram of Fig. $6(\mathrm{~b}), \omega \rightarrow \omega / \delta^{n}$, where $n$ is some rational number. The results are shown in Fig. 13. In particular we see that different values for $n$ are needed for the three different boundary lines in order to render them constant as a function of the horizontal axis, $\delta$. We find that the boundary between Region (i-a) and Regions (i-b-3), (i-b-6) (solid line in Fig. 6(b)) requires $n=3 / 2$, the boundary between Regions (i-b-2) and (i-b-3) requires $n=3 / 2$, the boundary between (i-b5 ) and (i-b-6) requires $n=1$, and the boundary between Regions (i-b-2),(i-b-5) and Regions (i-b-1),(i-b-4) (lower dashed line in Fig. 6(b)) requires $n=0$.

\section{ANALYSIS IN THE THOMAS-FERMI LIMIT}

\section{A. Zero (and low) rotation limit}

When the rotation frequency is zero or small, such that the number of vortices in either component is small, we 

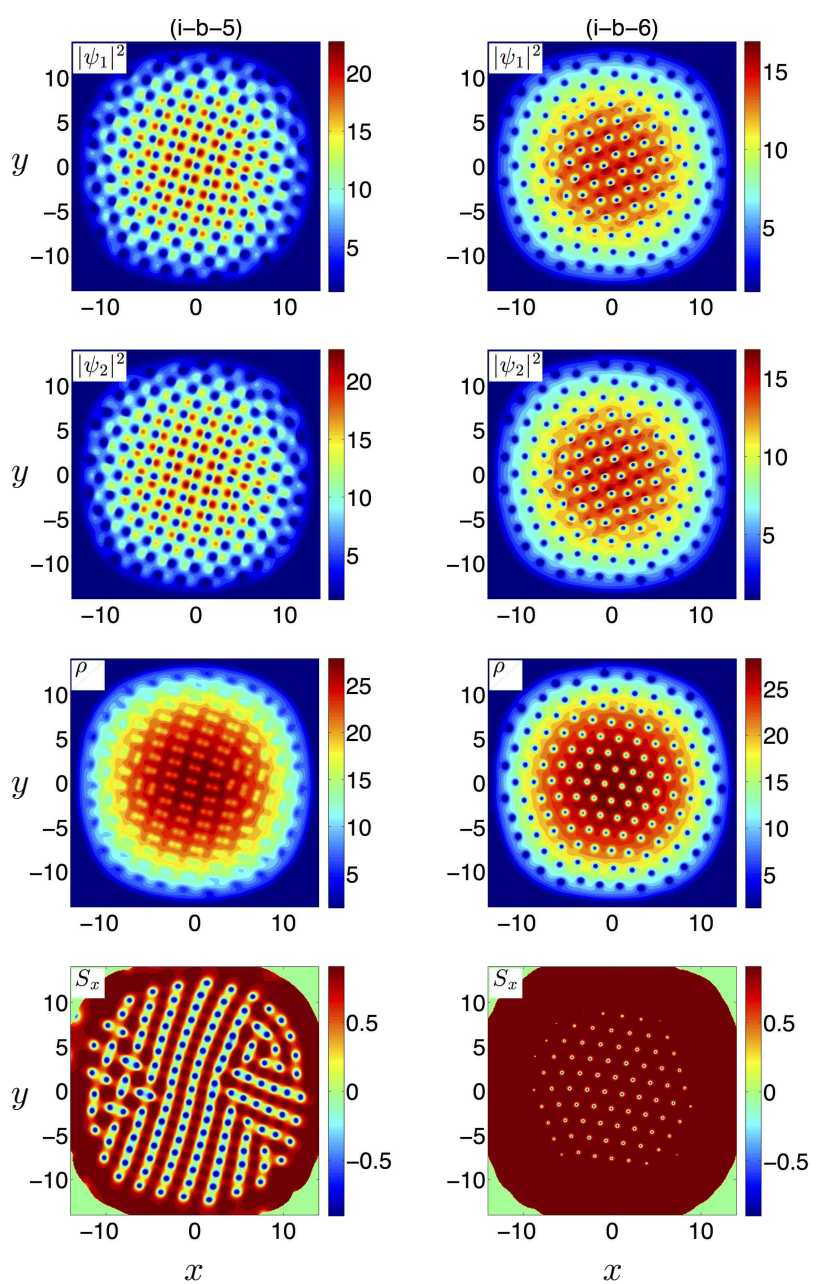

FIG. 10: (Color online) Example ground state profiles for regions (i-b-5) (left panels) and (i-b-6) (right panels) of the $\omega-\delta$ with $\Omega=0.9$ phase diagram of Fig. 6 showing $\left|\psi_{1}\right|^{2}$, $\left|\psi_{2}\right|^{2}, \rho$ and $S_{x}$. The profiles are taken at $(\delta, \omega)=(0.8,0.3)$ for region (i-b-5) and $(0.8,1.5)$ for region $(\mathrm{i}-\mathrm{b}-6)$. The densities are multiplied by $N$.
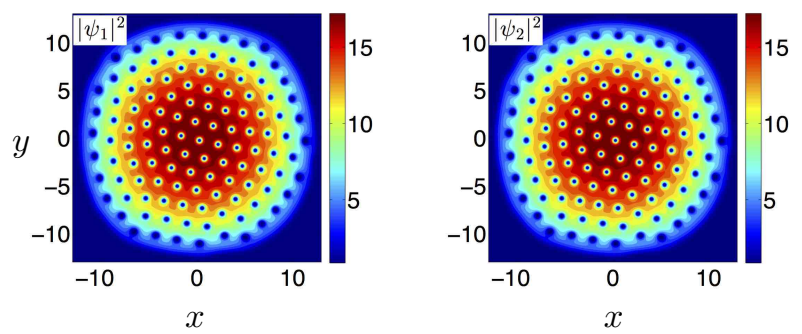

FIG. 11: (Color online) Example ground state density profiles for region (i-a) of the $\omega-\delta$ with $\Omega=0.9$ phase diagram of Fig. 6 . The profile is taken at $(\delta, \omega)=(0.2,6.75)$. The densities are multiplied by $N$.
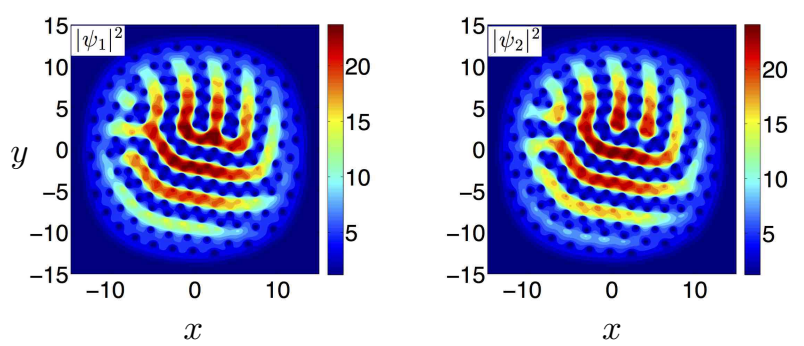

FIG. 12: (Color online) Example ground state density profiles for region (iii) of the $\omega-\delta$ with $\Omega=0.9$ phase diagram of Fig. 6. The profile is taken at $(\delta, \omega)=(1.2,2)$. The densities are multiplied by $N$.

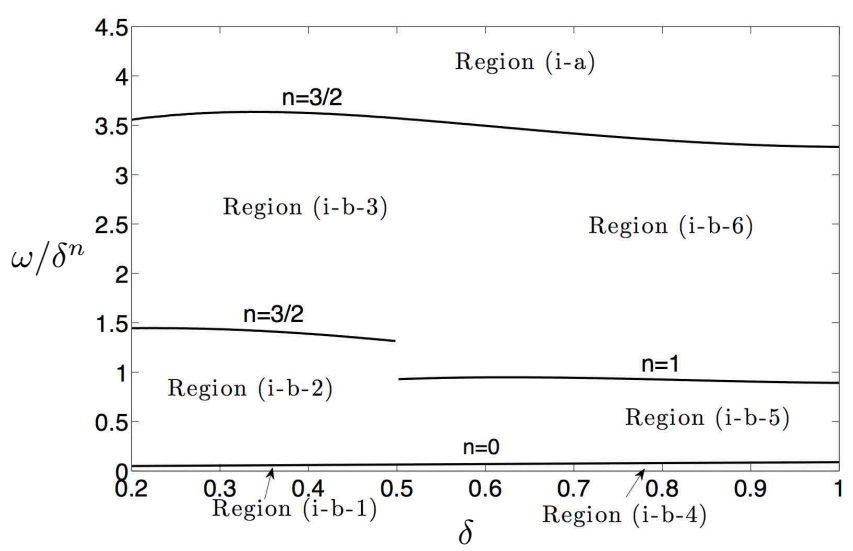

FIG. 13: $\omega / \delta^{n}-\delta$ phase diagram with $\Omega=0.9$. The numerical parameters are taken as $g=1$ and $N=10^{4}$, and the value of $n$ is indicated on the graph.

can use the Thomas-Fermi approximation for the density. This means that, in the energy functional (5), we can neglect $\left(\boldsymbol{v}_{\text {eff }}-\boldsymbol{\Omega} \times \boldsymbol{r}\right)^{2}\left[\boldsymbol{v}_{\text {eff }}\right.$ is defined in Eq. (4)] as well as the derivatives of $\rho$ and $\boldsymbol{S}$. This implies that we can neglect $S_{y}$, and we can define $S_{x}=\sin \phi$ and $S_{z}=\cos \phi$, such that the energy (5) reduces to

$$
\begin{aligned}
E_{T F}=\int & \frac{1}{2}\left(1-\Omega^{2}\right) r^{2} \rho+\frac{g N}{4}\left(2+(\delta-1) \sin ^{2} \phi\right) \rho^{2} \\
& -\rho \omega \sin \phi \quad d^{2} r .
\end{aligned}
$$

We will now examine this Thomas-Fermi energy in regions (i) and (ii) of the $\Omega=0$ and $\Omega=0.3$ phase diagrams (Figs. 1 and 3 ). Note that regions (i-a) and (i-b) present in the $\Omega=0.3$ phase diagram, are equivalent in the Thomas-Fermi limit.

To begin, we look to region (i) (including (i-a) and (ib)). In this case we have coincident disks meaning that $S_{z}=\cos \phi=0$ and therefore that $S_{x}=\sin \phi=1$. The energy then reduces to

$$
E=\int \frac{1}{2}\left(1-\Omega^{2}\right) r^{2} \rho+\frac{g N}{4}(\delta+1) \rho^{2}-\rho \omega \quad d^{2} r,
$$


which gives

$$
\frac{1}{2}\left(1-\Omega^{2}\right) r^{2}+\frac{g N}{2}(1+\delta) \rho-\omega=\mu,
$$

where $\mu$ is a Lagrange multiplier (chemical potential) to account for the conservation of the total number of particles. We set $\tilde{\mu}=\mu+\omega$, a constant, and use the normalisation condition $\int_{0}^{R_{\mathrm{TF}}} \rho=1$, to obtain expressions for the total density, the radius of the components, $R_{\mathrm{TF}}$, and the modified chemical potential $\tilde{\mu}$,

$$
\begin{aligned}
\rho & =\frac{2 \tilde{\mu}-\left(1-\Omega^{2}\right) r^{2}}{g N(1+\delta)}, \\
R_{\mathrm{TF}} & =\left(\frac{2 g N(1+\delta)}{\pi\left(1-\Omega^{2}\right)}\right)^{1 / 4}, \\
\tilde{\mu} & =\left(\frac{g N(1+\delta)\left(1-\Omega^{2}\right)}{2 \pi}\right)^{1 / 2} .
\end{aligned}
$$

In region (ii) we have non-coincident components. Figure 2(a) shows typical density profiles for the individual components and the total density. It is immediately clear that the profile of the individual components is divided into two sections. In the first section, which exists for $0<r<\tilde{r}$, the components are non-coincident, while in the second section, which exists for $\tilde{r}<r<R_{\mathrm{TF}}$, the components are coincident. Notice that all the while the total density remains an inverted parabola. We must therefore consider the full Thomas-Fermi energy (8). The resulting Euler-Lagrange equations are

$$
\begin{aligned}
\mu= & \frac{1}{2}\left(1-\Omega^{2}\right) r^{2}+\frac{g N}{2}\left(2+(\delta-1) \sin ^{2} \phi\right) \rho \\
& \quad-\omega \sin \phi, \\
0 & {\left[\frac{g N}{2}(\delta-1) \rho \sin \phi-\omega\right] \cos \phi, }
\end{aligned}
$$

which immediately gives that either $\cos \phi=0$ upon which we return to the above analysis to recover the expression for $\rho$ from Eq. (11a), or that $\cos \phi \neq 0$, in which case we get

$$
\rho=\frac{2 \omega}{g N(\delta-1) \sin \phi} .
$$

We now plug (13) into (12a) to obtain

$\mu=\frac{1}{2}\left(1-\Omega^{2}\right) r^{2}+\frac{\omega}{(\delta-1) \sin \phi}\left(2+(\delta-1) \sin ^{2} \phi\right)-\omega \sin \phi$,

which gives

$$
\begin{aligned}
\sin \phi & =\frac{2 \omega}{(\delta-1)\left(\mu-\frac{1}{2}\left(1-\Omega^{2}\right) r^{2}\right)}, \\
\rho & =\frac{\mu-\frac{1}{2}\left(1-\Omega^{2}\right) r^{2}}{g N} .
\end{aligned}
$$

Now we note that when $r=\tilde{r}$ we have $\sin \phi=1$, which gives

$$
\tilde{r}^{2}=\frac{2}{\left(1-\Omega^{2}\right)}\left(\mu+\frac{2 \omega}{(1-\delta)}\right)
$$

The next step is to complete the normalisation integral: this must be divided into the two noted sections, so that $1=\int_{0}^{\tilde{r}} \rho+\int_{\tilde{r}}^{R_{\mathrm{TF}}} \rho$, where the appropriate expression for $\rho$ is given by Eq. (15b) for the first integral and by Eq. (11a) for the second integral. Using that $\rho\left(R_{\mathrm{TF}}\right)=0$, that is $2(\mu+\omega)=\left(1-\Omega^{2}\right) R_{\mathrm{TF}}^{2}$, and that at $r=\tilde{r}$, $\sin \phi=1$, and thus

$$
\frac{2 \omega(\delta+1)}{(\delta-1)}=\left(1-\Omega^{2}\right)\left(R_{\mathrm{TF}}^{2}-\tilde{r}^{2}\right)
$$

we then get

$$
\begin{aligned}
R_{\mathrm{TF}} & =\left[\frac{2}{\left(1-\Omega^{2}\right)}\left(\omega+\sqrt{\left.\frac{2 \omega^{2}}{(1-\delta)}+\frac{g N\left(1-\Omega^{2}\right)}{\pi}\right)}\right]_{(17 \mathrm{a},}^{1 / 2}\right. \\
\mu & =\sqrt{-\frac{2 \omega^{2}}{(\delta-1)}+\frac{g N\left(1-\Omega^{2}\right)}{\pi}} .
\end{aligned}
$$

With these expressions we are able to rewrite $\tilde{r}$ such that

$$
\tilde{r}^{2}=R_{T F}^{2}-\frac{2 \omega(1+\delta)}{(\delta-1)\left(1-\Omega^{2}\right)},
$$

i.e. provided we satisfy the original assumption that $\delta>$ 1 , this expression remains less than $R_{T F}{ }^{2}$, except when $\omega=0$.

We plot these analytical expressions for the total density and the spin density components $S_{x}$ and $S_{z}$ in an example case for region (ii); see Fig. 2. Comparison with the numerical simulations in this case, and throughout region (ii), is good. We can also define more precisely the profile of the individual wave functions near the centre of the condensate, as well as in the limits of $\delta$ large and $\omega$ small. The wave functions are composed through $\left|\psi_{1,2}\right|^{2}=\rho(1 \pm \cos \phi) / 2$, such that in the region $r<\tilde{r}$, $\rho$ and $\cos \phi$ can found from Eq. (15). At the origin $\rho(0)=\mu /(2 g N)$, and

$$
\begin{aligned}
S_{z}(0) & =\sqrt{1-\frac{4 \omega^{2}}{(\delta-1)^{2} \mu^{2}}} \\
& \approx 1-\frac{2 \omega^{2}}{(\delta-1)^{2} \mu^{2}},
\end{aligned}
$$

giving

$$
\begin{aligned}
\left|\psi_{1}(0)\right|^{2} & \approx \frac{\omega^{2}}{g N(\delta-1)^{2} \mu} \\
\left|\psi_{2}(0)\right|^{2} & \approx \frac{\mu}{g N}\left(1-\frac{\omega^{2}}{(\delta-1)^{2} \mu^{2}}\right) .
\end{aligned}
$$


The profiles for both individual components as well as the total density are plotted alongside the numerical simulations in Fig. 2(a). Note that when $r>\tilde{r}, \cos \phi=0$ and hence $\left|\psi_{1}\right|^{2}=\left|\psi_{2}\right|^{2}=\rho / 2$, with $\rho$ given by Eq. (11a).

Finally, we are left to calculate the boundary between regions (i) and (ii). This occurs when $\tilde{r} \rightarrow 0$ which implies that $\omega \rightarrow \omega_{c}$, where

$$
\omega_{c}=\frac{(\delta-1)}{(1+\delta)^{1 / 2}}\left(\frac{g N\left(1-\Omega^{2}\right)}{2 \pi}\right)^{1 / 2} .
$$

We plot this critical Rabi frequency as a function of $\delta$ on both the $\Omega=0$ and $\Omega=0.3$ phase diagrams of Figs. 1 and 3 (dotted lines). The agreement in both cases is good.

\section{B. Estimate of the Rabi Energy}

One striking feature of the Rabi-coupled condensates is the nature of vortex-vortex interaction between vortices in different components. In particular, the formation of the vortex molecule, whereby two like-signed vortices, one from each component, bind together, plays a fundamental role in the vortex lattices pictures that we have previously described in Sect. III. The Thomas-Fermi approximation will provide us with the necessary framework in which we will be able to estimate the energy

$$
E_{\mathrm{rabi}}=-\omega \int \psi_{1}^{*} \psi_{2}+\psi_{1} \psi_{2}^{*}
$$

We will see that if $p_{i}$ are the vortex positions in component 1 , and $q_{j}$ in component 2 , then the Rabi energy can be estimated to be proportional to

$$
\sum_{i, j}\left|p_{i}-q_{j}\right|^{2}\left(d_{1}+d_{2} \ln \left|p_{i}-q_{j}\right|^{2}\right)+d_{3} \sum_{i, j} \ln \left|p_{i}-q_{j}\right|^{2}
$$

where $d_{1}, d_{2}, d_{3}$ are proportional to $\omega$ and will be precisely estimated. The analysis will follow closely that described in our previous work, namely finding energy estimates for the case of a rotating two-component condensate [15]: here we give a brief overview of that analysis before quoting the results. This, together with an estimate for the Rabi energy above (22), which we also detail below, gives a complete estimation for the full energy of the system considered in this paper.

We begin with the full energy defined in Eq. (1), making the rescaling $\psi_{k}(x, y)=\epsilon u_{k}(x \sqrt{\epsilon}, y \sqrt{\epsilon})$, where $\epsilon=1 / \sqrt{N}$ is small, to get

$$
\begin{aligned}
E_{\Omega, \omega}\left(u_{1}, u_{2}\right)= & \int \sum_{k=1}^{2}\left(\frac{\epsilon^{2}}{2}\left|\nabla u_{k}\right|^{2}+\frac{1}{2} r^{2}\left|u_{k}\right|^{2}\right. \\
& \left.-\epsilon(\Omega \times \boldsymbol{r}) \cdot\left(i u_{k}, \nabla u_{k}\right)+\frac{g}{2}\left|u_{k}\right|^{4}\right) \\
& +g_{12}\left|u_{1}\right|^{2}\left|u_{2}\right|^{2}-\epsilon \omega\left(u_{1}^{*} u_{2}+u_{1} u_{2}^{*}\right) \quad d^{2} r,
\end{aligned}
$$

where $(i u, \nabla u)=i u \nabla u^{*}-i u^{*} \nabla u$. The energy splitting analysis detailed in Ref. [15] amounts to writing the complex functions $u_{k}$ as products of the real ground state at $\Omega=0$ times the vortex contribution. Thus we write $u_{k}=\eta f_{k}$, where the $\eta$ is the ground state solutions for $\Omega=0$, and where the $f_{k}$ are functions that include the vortex-peak profiles. Away from these vortex-peaks, we expect the $f_{k}$ to be almost equal to 1 . Substitution of these forms for the $u_{k}$ into the above energy, and after a few simple algebraic steps, allows us to re-express the energy as $E_{\Omega, \omega}\left(u_{1}, u_{2}\right)=E_{0, \omega}(\eta, \eta)+F_{\Omega, \omega}\left(f_{1}, f_{2}\right)$, where

$$
\begin{aligned}
F_{\Omega, \omega}\left(f_{1}, f_{2}\right)=\int \sum_{k=1}^{2}\left(\frac{\epsilon^{2}}{2} \eta^{2}\left|\nabla f_{k}\right|^{2}+\frac{1}{2} g \eta^{4}\left(\left|f_{k}\right|^{2}-1\right)^{2}\right. \\
\left.\quad-\epsilon \eta^{2}(\Omega \times \boldsymbol{r}) \cdot\left(i f_{k}, \nabla f_{k}\right)\right) \\
+g_{12} \eta^{2} \eta^{2}\left(1-\left|f_{1}\right|^{2}\right)\left(1-\left|f_{2}\right|^{2}\right) \\
+\epsilon \omega \eta^{2}\left|f_{2}-f_{1}\right|^{2} \quad d^{2} r .
\end{aligned}
$$

We note that the trapping potential does not appear in the above expression for the energy $F_{\Omega, \omega}$; it is confined to $E_{0, \omega}$.

The energy $E_{\Omega, \omega}\left(u_{1}, u_{2}\right)$ is exactly the same as that of a rotating two-component condensate with the addition of a Rabi coupling. The case of $F_{\Omega, 0}$ was considered in Ref. [15] where it is approximated in terms of $p_{i}$ and $q_{j}$, the locations of vortices in component 1 and component 2 respectively:

$$
\begin{aligned}
E_{\omega=0}^{p v}= & \frac{1}{2} \pi \epsilon^{2} \eta^{2}(0)\left[-\sum_{i \neq j} \ln \left|p_{i}-p_{j}\right|^{2}-\sum_{i \neq j} \ln \left|q_{i}-q_{j}\right|^{2}\right. \\
& +\Omega \sum_{i}\left|p_{i}\right|^{2}+\Omega \sum_{i}\left|q_{i}\right|^{2} \\
& \left.+\frac{2 \sqrt{\pi(1+\delta)} \delta \epsilon|\ln \epsilon|}{\left(1-\delta^{2}\right)} \sum_{i, j} \frac{1}{\left|p_{i}-q_{j}\right|^{2}}\right] .
\end{aligned}
$$

From [15], we recall that $\eta^{2}(0)=(\pi g(1+\delta))^{-1 / 2}$. The core behaviour around a vortex-peak is given by $\left(v_{1}(r) e^{i \theta}, v_{2}(r)\right)$ where $v_{1}$ and $v_{2}$ solve the following sys- 
tem

$$
\begin{aligned}
& -\frac{\left(r v_{1}^{\prime}\right)^{\prime}}{r}+\frac{v_{1}}{r^{2}}+2 \eta^{2}(0)\left(v_{1}^{2}-1\right) v_{1} \\
& +2 g_{12} \eta^{2}(0) v_{1}\left(v_{2}^{2}-1\right)=0 \\
& -\frac{\left(r v_{2}^{\prime}\right)^{\prime}}{r}+2 \eta^{2}(0)\left(v_{2}^{2}-1\right) v_{2} \\
& +2 g_{12} \eta^{2}(0) v_{2}\left(v_{1}^{2}-1\right)=0
\end{aligned}
$$

From this system, asymptotic expansions can be obtained for $v_{1}$ and $v_{2}$ at infinity: $v_{1}(r)-1 \sim-\gamma_{1} / r^{2}$ and $v_{2}(r)-$ $1 \sim \gamma_{2} / r^{2}$ for some constants $\gamma_{1}$ and $\gamma_{2}$,

$$
\gamma_{1}=\frac{1}{4 \eta^{2}(0) g\left(1-\delta^{2}\right)}, \quad \gamma_{2}=\frac{g_{12}}{4 \eta^{2}(0) g^{2}\left(1-\delta^{2}\right)} .
$$

A vortex-peak in each component dictates that the wave function in component 1 behaves like $v_{1}(|x-p|) e^{i \theta} v_{2}(\mid x-$ $q \mid$ ) where $p$ is a vortex in component 1 and $q$ is a vortex in component 2 , or equivalently a peak in component 1 . We wish to calculate the contribution of the Rabi energy

$$
F_{\Omega, \omega}-F_{\Omega, 0}=\epsilon \omega \int \eta^{2}\left|f_{2}-f_{1}\right|^{2} .
$$

Therefore, we take $f_{k}=\nu_{k} e^{i \theta_{k}}$, for phases $\theta_{k}$ and vortexpeak profile functions, $\nu_{k}$, given as

$$
\nu_{k}=\left(1-\gamma_{1}\left(\frac{\epsilon}{r_{k}}\right)^{2}\right)\left(1+\gamma_{2}\left(\frac{\epsilon}{r_{3-k}}\right)^{2}\right)+O\left(\frac{\epsilon}{r}\right)^{4},
$$

where we have rescaled around the vortex core so that the vortex in component $k$ is defined to be at $r_{k} / \epsilon$, while the peak is at $r_{3-k} / \epsilon$. This means that

$$
\begin{aligned}
\left|f_{2}-f_{1}\right|^{2}= & v_{1}^{2}+v_{2}^{2}-2 v_{1} v_{2} \cos \left(\theta_{1}-\theta_{2}\right) \\
= & 2\left(1-\cos \left(\theta_{1}-\theta_{2}\right)\right) \\
& +\frac{\epsilon^{2}\left(r_{1}^{2}+r_{2}^{2}\right)}{r_{1}^{2} r_{2}^{2}}\left(\gamma_{2}-\gamma_{1}\right)\left(1-\cos \left(\theta_{1}-\theta_{2}\right)\right) \\
& +O\left(\frac{\epsilon}{r}\right)^{4} .
\end{aligned}
$$

We recall that

$$
\gamma_{2}-\gamma_{1}=-\frac{1}{4} \sqrt{\frac{\pi}{g(1+\delta)}}
$$

From now on, we will work in the rescaled distance, $\tilde{r}=r / \epsilon$, and drop the tilde notation. We first deal with the leading order term, $I_{0}=2 \epsilon \omega \int \eta^{2}\left(1-\cos \left(\theta_{1}-\theta_{2}\right)\right)$. Basic geometric arguments (see for example [21]) for a pair of vortex-peaks with the vortex in component $1(-2)$ located at $r=R(-R)$ give that

$$
\cos \left(\theta_{1}-\theta_{2}\right)= \pm \sqrt{\frac{A+B}{A+B \cos (2 \theta)}},
$$

where $A=r^{4}+R^{4}$ and $B=-2 r^{2} R^{2}$ and with a positive sign allocated if $r^{2}>R^{2}$ and a negative sign if $r^{2}<R^{2}$. Then

$$
\begin{aligned}
\int_{0}^{2 \pi} \cos \left(\theta_{1}-\theta_{2}\right) & = \pm \int_{0}^{2 \pi} \sqrt{\frac{A+B}{A+B \cos (2 \theta)}} \\
& = \pm 4 K(1-\beta)
\end{aligned}
$$

with $K\left(k^{2}\right)$ the complete elliptical integral of the 1st kind defined by

$$
K\left(k^{2}\right)=\int_{0}^{1} \frac{d t}{\left(1-t^{2}\right)\left(1-k^{2} t^{2}\right)},
$$

and where $\beta=(A-B) /(A+B)$, which means that $1-\beta=-4 r^{2} R^{2} /\left(r^{2}-R^{2}\right)^{2}$. We now perform a rescaling, writing $u=r^{2} / R^{2}$, and bring the $\eta$ out of the integral. This leads to

$$
\begin{aligned}
& I_{0} \sim 2 \pi \epsilon \omega R^{2} \eta^{2}(0) \times \\
& \quad\left[\int_{0}^{1} 1+\frac{2}{\pi} K\left(-\frac{4 u}{(u-1)^{2}}\right) d u\right. \\
& \left.\quad+\int_{1}^{\left(\frac{R_{T F}}{R}\right)^{2}} 1-\frac{2}{\pi} K\left(-\frac{4 u}{(u-1)^{2}}\right) d u\right]
\end{aligned}
$$

where we have split the integral to account for the singularity at $u=1$ (equivalently $r= \pm R$ ). We now proceed by expanding the elliptic integral, which gives for small argument, $K\left(k^{2}\right) \sim \pi / 2+\pi / 8 k^{2}+\ldots$ Thus we can write

$$
\begin{aligned}
\int_{1}^{\left(\frac{R_{\mathrm{TF}}}{R}\right)^{2}} 1-\frac{2}{\pi} K\left(-\frac{4 u}{(u-1)^{2}}\right) d u \\
=\int_{1}^{r^{\prime}} 1-\frac{2}{\pi} K\left(-\frac{4 u}{(u-1)^{2}}\right) d u \\
\quad+\int_{r^{\prime}}^{\left(\frac{R_{\mathrm{TF}}^{R}}{R}\right)^{2}} 1-\frac{2}{\pi} K\left(-\frac{4 u}{(u-1)^{2}}\right) d u \\
\sim \int_{1}^{r^{\prime}} 1-\frac{2}{\pi} K\left(-\frac{4 u}{(u-1)^{2}}\right) d u \\
\quad+\int_{r^{\prime}}^{\left(\frac{R_{\mathrm{TF}}^{R}}{R}\right)^{2}} \frac{1}{u-1}+\frac{1}{(u-1)^{2}} d u \\
\sim \int_{1}^{r^{\prime}} 1-\frac{2}{\pi} K\left(-\frac{4 u}{(u-1)^{2}}\right) d u \\
\quad+\ln \left(\frac{R_{\mathrm{TF}}}{R}\right)^{2}-\ln \left(r^{\prime}-1\right)+\frac{1}{r^{\prime}-1},
\end{aligned}
$$


with $r^{\prime}$ chosen such that the expansion of the elliptic integral up to first order is valid and where in the last line we have assumed that $R_{\mathrm{TF}} \gg R$. This then gives

$$
I_{0} \sim 2 \pi \epsilon \omega R^{2} \eta^{2}(0)\left[\tilde{d}_{1}-\ln \left(R^{2}\right)\right]
$$

where

$$
\begin{aligned}
\tilde{d}_{1}= & \int_{0}^{1} 1+\frac{2}{\pi} K\left(-\frac{4 u}{(u-1)^{2}}\right) \quad d u \\
& +\int_{1}^{r^{\prime}} 1-\frac{2}{\pi} K\left(-\frac{4 u}{(u-1)^{2}}\right) \quad d u \\
& +\ln \left(\frac{R_{\mathrm{TF}}^{2}}{r^{\prime}-1}\right)+\frac{1}{r^{\prime}-1} \\
\sim & 5.95,
\end{aligned}
$$

being to this level of approximation independent of $r^{\prime}$. Similar arguments for the next order energy (using Eq. (31) in Eq. (29)) give

$$
I_{1} \sim 4 \pi \epsilon^{3} \omega\left(\gamma_{2}-\gamma_{1}\right) \eta^{2}(0) \ln \left(R^{2}\right)+\text { constant. }
$$

Putting this altogether gives our estimate for the Rabi energy as

$$
2 \pi \epsilon \omega \eta_{1}(0) \eta_{2}(0)\left[\left(\tilde{d}_{1}-\ln R^{2}\right) R^{2}+2 \epsilon^{2}\left(\gamma_{2}-\gamma_{1}\right) \ln \left(R^{2}\right)\right] .
$$

Equation (41) is the contribution of the Rabi terms to the point energy. We now note that $2 R=\left|p_{i}-q_{j}\right|$ and rescale the vortex positions as $p_{i}=\sqrt{\epsilon} \tilde{p}_{i}, q_{j}=\sqrt{\epsilon} \tilde{q}_{j}$ to render it in the correct scaling of Eq. (26). Combining (41) with the non-Rabi point energy Eq. (26) gives a point energy that the $p_{i}$ and $q_{j}$ minimise, namely

$$
\begin{aligned}
E^{p v}= & \frac{1}{2} \pi \epsilon^{2} \eta^{2}(0)\left[-\sum_{i \neq j} \ln \left|p_{i}-p_{j}\right|^{2}-\sum_{i \neq j} \ln \left|q_{i}-q_{j}\right|^{2}\right. \\
& +\Omega \sum_{i}\left|p_{i}\right|^{2}+\Omega \sum_{i}\left|q_{i}\right|^{2}+c_{1} \sum_{i, j} \frac{1}{\left|p_{i}-q_{j}\right|^{2}} \\
& +d_{1} \sum_{i, j}\left|p_{i}-q_{j}\right|^{2}+d_{2} \sum_{i, j}\left|p_{i}-q_{j}\right|^{2} \ln \left|p_{i}-q_{j}\right|^{2} \\
& \left.+d_{3} \sum_{i, j} \ln \left|p_{i}-q_{j}\right|^{2}\right],
\end{aligned}
$$
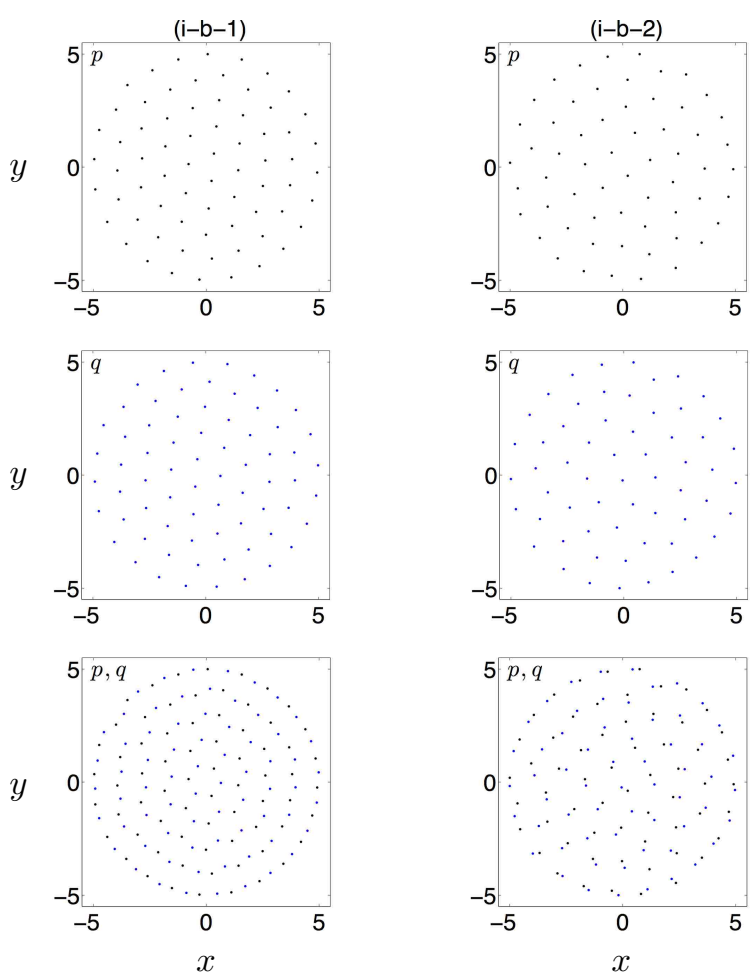

FIG. 14: (Color online) Vortex lattice profiles for the two components as numerically simulated through the point vortex energy of Eq. (42). Shown are the results for regions (i-b-1) [left panels] and (i-b-2) [right panels], for the $p_{i}$ (component 1: black dots), $q_{i}$ (component 2: blue dots) and both component 1 and 2 together. The parameters are chosen as $\Omega=0.9$, with $c_{1}=0.006, d_{1}=0.0001, d_{2}=0$ and $d_{3}=-0.001$ for region (i-b-1) and $c_{1}=0.006, d_{1}=0.0225, d_{2}=-0.0019$ and $d_{3}=-0.0975$ for region (i-b-2).

where

$$
\begin{aligned}
& c_{1}=\frac{2 \sqrt{\pi(1+\delta)} \delta \epsilon|\ln \epsilon|}{\left(1-\delta^{2}\right)}, \\
& d_{1}=\omega\left(\tilde{d}_{1}-\ln (\epsilon / 4)\right), \\
& d_{2}=-\omega \\
& d_{3}=-2 \omega \epsilon \sqrt{\frac{\pi}{g(1+\delta)}} .
\end{aligned}
$$

We note that higher order expansion of the Rabi energy produces terms like $\epsilon^{2} \omega|\ln \epsilon| /\left|p_{i}-q_{j}\right|^{2}$, which provides a small $\epsilon \omega$ correction to the $c_{1} /\left|p_{i}-q_{j}\right|^{2}$ term, and can be safely ignored.

Minimization of the point vortex energy (42) recreates the vortex lattice configurations that we see in regions (ib-1) through (i-b-6) in the phase diagram of Fig. 6 and in Figs. 7-10. Our results are shown in Figs. 14 and 15. 

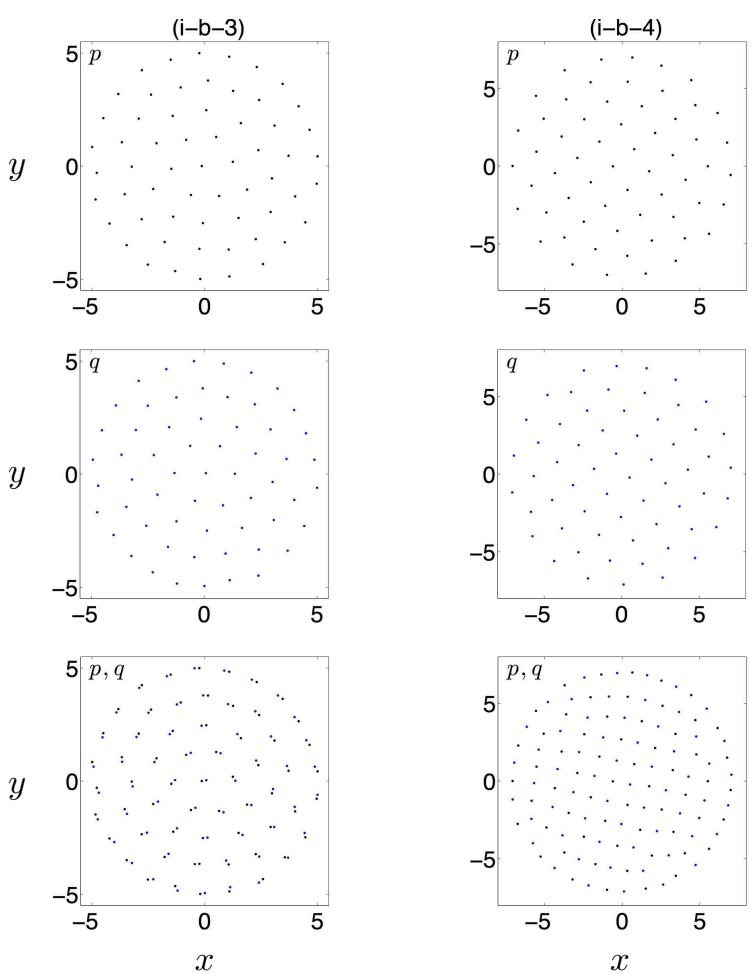

FIG. 15: (Color online) Vortex lattice profiles for the two components as numerically simulated through the point vortex energy of Eq. (42). Shown are the results for regions (i-b-1) [left panels] and (i-b-2) [right panels], for the $p_{i}$ (component 1: black dots), $q_{i}$ (component 2: blue dots) and both component 1 and 2 together. The parameters are chosen as $\Omega=0.9$, with $c_{1}=0.006, d_{1}=0.09, d_{2}=-0.0075$ and $d_{3}=-0.39$ for region (i-b-3) and $c_{1}=0.124, d_{1}=0.06$, $d_{2}=0$ and $d_{3}=-0.005$ for region (i-b-4).

\section{Vortex Molecules: Separation of vortices}

We now turn to the issue of the properties of an individual vortex molecule, that is to say we consider a single vortex-peak in each component, with the vortex (peak) in component $k$ is defined to be at $+R(-R)$. This makes the separation of the two vortices to be equal to $2 R$. Our aim is to establish the dependence of the separation on the strength of the Rabi coupling $\omega$. There are three terms whose effect we need to consider: the rotation, the two-body coupling and the Rabi coupling; in much the same vein as above these give the following energetic expressions

$$
\begin{aligned}
J_{\Omega} & =\Omega R^{2} \\
J_{g_{12}} & =\frac{\epsilon^{3} \sqrt{\pi(1+\delta)} \delta|\ln \epsilon|}{4\left(1-\delta^{2}\right)} \frac{1}{R^{2}} \\
J_{\omega} & =2 \omega\left[\left(\tilde{d}_{1}-\ln R^{2}\right) R^{2}+2 \epsilon^{2}\left(\gamma_{2}-\gamma_{1}\right) \ln R^{2}\right]
\end{aligned}
$$

The total energy we need to consider is thus $J=J_{\Omega}+$

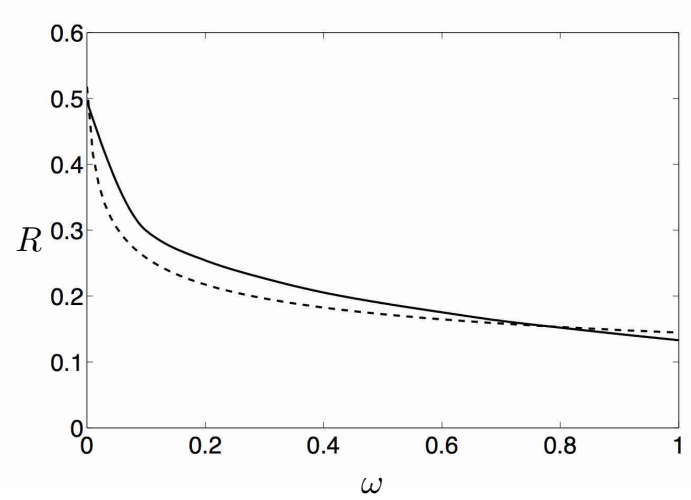

FIG. 16: A plot of half the separation $R$ of two vortex-peaks as a function of the Rabi coupling $\omega$ showing a comparison between the numerics (solid line) and analytics of Eq. (48) (dashed line). The vortex-peak in component $1(2)$ is positioned at $+R(-R)$. The values chosen are: $\Omega=0.15, \delta=0.4$, $g=1$ and $\epsilon=0.01$.

$J_{g_{12}}+J_{\omega}$, for which we must revert back to the original scaling through $R=\sqrt{\epsilon} \tilde{R}$. This gives (extracting an $\epsilon$ factor and dropping tildes)

$$
\begin{aligned}
J=R^{2} & \left(\Omega+2 \omega\left(\tilde{d}_{1}+|\ln \epsilon|\right)\right) \\
& -2 \omega R^{2} \ln R^{2}+4 \epsilon \omega\left(\gamma_{2}-\gamma_{1}\right) \ln R^{2} \\
& +\frac{\epsilon \sqrt{\pi(1+\delta)} \delta|\ln \epsilon|}{4\left(1-\delta^{2}\right)} \frac{1}{R^{2}}
\end{aligned}
$$

from which we differentiate with respect to $R$ to obtain the optimum vortex separation; this gives us the following expression

$$
\begin{aligned}
& \left(\Omega+2 \omega\left(\tilde{d}_{1}+|\ln \epsilon|\right)\right)-2 \omega\left(1+\ln R^{2}\right) \\
& \quad+4 \epsilon \omega\left(\gamma_{2}-\gamma_{1}\right) \frac{1}{R^{2}}-\frac{\epsilon \sqrt{\pi(1+\delta)} \delta|\ln \epsilon|}{4\left(1-\delta^{2}\right)} \frac{1}{R^{4}}=(048)
\end{aligned}
$$

In particular, if $\omega=0$, then this expression simplifies to

$$
R=\left(\frac{\epsilon \sqrt{\pi(1+\delta)} \delta|\ln \epsilon|}{4 \Omega\left(1-\delta^{2}\right)}\right)^{\frac{1}{4}}, \quad \text { for } R \neq 0
$$

In Fig. 16 we plot a comparison between the above relationship Eq. (48) and numerical simulations, focussing on the case when $\Omega=0.15$ (thus permitting only a single vortex molecule), $\delta=0.4, g=1$ and $\epsilon=0.01$. We can see a good agreement between the numerical curve and the estimate. 


\section{CONCLUSION}

We have classified the ground states of the energy describing rotating two-component Bose-Einstein condensates under the effect of internal coherent Rabi coupling, according to the magnitude of the Rabi coupling, the magnitude of the intercomponent coupling and the rotational velocity. We have found numerically, and in some cases analytically, the boundaries between different patterns, namely symmetry breaking components and various vortex patterns. We estimate the Rabi energy in the Thomas-Fermi limit which allows us to have an analytical description of the point energy governing the location of vortices. The specificity of two-component condensates is that a vortex in one component induces a peak in the other. It is the interaction between vortices and peaks which create the different shapes of lattices: hexagonal, triangular, square, by pairs, or well separated. We also analyze the formation of a vortex molecule made up of two vortex-peaks according to the magnitude of the Rabi coupling.

\section{ACKNOWLEDGEMENTS}

This work was supported by a public grant as part of the Investissement d'avenir project, reference ANR-11LABX-0056-LMH, LabEx LMH.

[1] C.J. Myatt, E.A. Burt, R.W. Ghrist, E.A. Cornell, and C.E. Wieman, Phys. Rev. Lett. 78, 586-589 (1997).

[2] D.M. Stamper-Kurn et al., Phys. Rev. Lett. 80, 20272030 (1998).

[3] D.S. Hall, M.R. Matthews, J.R. Ensher, C.E. Wieman, and E.A. Cornell, Phys. Rev. Lett. 81, 1539-1542 (1998).

[4] D.M. Stamper-Kurn et al., Phys. Rev. Lett. 83, 661-664 (1999).
[5] M. R. Matthews et al., Phys. Rev. Lett. 83, 3358-3361 (1999).

[6] D. M. Stamper-Kurn and M. Ueda, Rev. Mod. Phys. 85, 1191 (2013).

[7] S. Seo et al., Phys. Rev. Lett. 115, 015301 (2015).

[8] M. R. Matthews et al., Phys. Rev. Lett. 83, 2498-2501 (1999).

[9] V. Schweikhard et al., Phys. Rev. Lett. 93, 210403 (2004).

[10] T.-L. Ho, Phys. Rev. Lett. 81, 742-745 (1998).

[11] T. Ohmi and K. Machida, J. Phys. Soc. Jpn. 67, 18221825 (1998).

[12] C.K. Law, H. Pu and N.P. Bigelow, Phys. Rev. Lett. 81, 5257-5261 (1998).

[13] E. J. Mueller and T-L. Ho, Phys. Rev. Lett. 88, 180403 (2002).

[14] P. Mason and A. Aftalion, Phys. Rev. A 84, 033611 (2011).

[15] A. Aftalion, P. Mason and J. Wei, Phys. Rev. A 85, 033614 (2012)

[16] N. Ghazanfari, A. Keles, and M.Ö. Oktel, Phys. Rev. A 89, 025601, 2014.

[17] W. E. Shirley, B. M. Anderson, C. W. Clark, and R. M. Wilson, Phys. Rev. Lett. 113, 165301 (2014).

[18] X.-F. Zhou, Z.-W. Zhou, C. Wu, and G.-C. Guo, Phys. Rev. A 91, 033603 (2015).

[19] P. Kuopanportti, N. V. Orlova, M. V. Milosevic, Phys. Rev. A 91, 043605 (2015).

[20] K. Kasamatsu, M. Tsubota, and M. Ueda, Phys. Rev. A 71, 043611 (2005).

[21] M. Eto, K. Kasamatsu, M. Nitta, H. Takeuchi, and M. Tsubota, Phys. Rev. A. 83, 063603 (2011).

[22] K. Kasamatsu, M.Eto, M. Nitta, Phys. Rev. A 93, 013615 (2016)

[23] M. Kobayashi, M. Nitta, Phys. Rev. D 87, 125013 (2013).

[24] M. Cipriani and M. Nitta, Phys. Rev. Lett. 111, 170401 (2013).

[25] M. Kobayashi, M. Nitta, J. Low Temp. Phys. 175, 208215 (2014).

[26] S. Lellouch, T.-L. Dao, T. Koffel, and L. SanchezPalencia, Phys. Rev. A 88, 063646 (2013).

[27] K. Kasamatsu and M. Tsubota, Phys. Rev. A 79, 023606 (2009). 